\title{
Cerebrospinal Fluid Macrophage Biomarkers in Amyotrophic Lateral Sclerosis
}

\author{
Alexander G. Thompson, BA, BMBCh $\mathbb{0}^{1}{ }^{1 *}$ Elizabeth Gray, BSc, PhD, ${ }^{1 *}$ \\ Marie-Laëtitia Thézénas, $M S_{1}{ }^{2}$ Philip D. Charles, MA, MSc, ${ }^{2}$ \\ Samuel Evetts, BSc, MSc, ${ }^{1}$ Michele T. Hu, MBBS, PhD, ${ }^{1}$ \\ Kevin Talbot, MBBS, DPhil, ${ }^{1}$ Roman Fischer, PhD, ${ }^{2}$ \\ Benedikt M. Kessler, BM, $\mathrm{PhD}^{2}$ and Martin R. Turner, MA, MBBS, PhD (1)
}

\begin{abstract}
Objective: The neurodegenerative disease, amyotrophic lateral sclerosis (ALS), is a heterogeneous clinical syndrome involving multiple molecular pathways. The development of biomarkers for use in therapeutic trials is a priority. We sought to use a high-throughput proteomic method to identify novel biomarkers in individual cerebrospinal fluid (CSF) samples.

Methods: Liquid chromatography/tandem mass spectrometry with label-free quantification was used to identify CSF proteins using samples from a well-characterized longitudinal cohort comprising patients with ALS $(n=43)$, the upper motor neuron variant, primary lateral sclerosis (PLS; $n=6)$, and cross-sectional healthy $(n=20)$ and disease controls (Parkinsons' disease, $\mathrm{n}=20$; ALS mimic disorders, $\mathrm{n}=12$ ).

Results: Three macrophage-derived chitinases showed increased abundance in ALS: chitotriosidase (CHIT1), chitinase-3-like protein 1 (CHI3L1), and chitinase-3-like protein 2 (CHI3L2). Elevated CHI3L1 was common to ALS and PLS, whereas CHIT1 and CHI3L2 levels differed. Chitinase levels correlated with disease progression rate (CHIT1, $r=$ $0.56, p<0.001 ; \mathrm{CHI} 3 \mathrm{~L} 1, r=0.31 ; p=0.028 ; \mathrm{CHI} 3 \mathrm{~L} 2, r=0.29, p=0.044)$. CHIT1, CHI3L1, and CHI3L2 levels correlated with phosphorylated neurofilament heavy chain ( $\mathrm{pNFH} ; r=0.62, p<0.001 ; r=0.49, p<0.001 ; r=0.41, p<$ 0.001). CHI3L1 levels, but not CHIT1 or CHI3L2, increased over time in those with low initial levels (gradient $=0.005$ $\log$ abundance units/month, $p=0.001$ ). High CHIT1 was associated with shortened survival (hazard ratio [HR] 2.84; $p=0.009$ ). Inclusion of pNFH in survival models left only an association of $p N F H$ and survival (HR 1.26; $p=0.019$ ).

Interpretation: Neuroinflammatory mechanisms have been consistently implicated through various experimental paradigms. These results support a key role for macrophage activity in ALS pathogenesis, offering novel target engagement and pharmacodynamic biomarkers for neuroinflammation-focused ALS therapy.
\end{abstract}

ANN NEUROL 2018;83:258-268

$\Lambda$ myotrophic lateral sclerosis (ALS; the commonest phenotype of motor neuron disease $[\mathrm{MND}]$ ) is a fatal adult-onset neurodegenerative disease characterized by progressive muscle weakness attributed to variable loss of upper motor neurons of the motor cortex and corticospinal tract and their lower motor neuron brainstem nuclear and spinal cord anterior horn connections. ALS has clinical, pathological, and genetic overlap with frontotemporal dementia (FTD). Death typically occurs within 3 years of symptom onset, attrubuted to respiratory failure, though survival varies widely from a few months up to decades. ${ }^{1}$ Over $95 \%$ of cases are associated

View this article online at wileyonlinelibrary.com. DOI: 10.1002/ana.25143

Received Aug 15, 2017, and in revised form Jan 4, 2018. Accepted for publication Jan 9, 2018.

Address correspondence to Prof Martin Turner, Clinical Neurosciences, West Wing Level 6, John Radcliffe Hospital, Oxford, OX3 9DU, United Kingdom. E-mail: martin.turner@ndcn.ox.ac.uk; or Prof Benedikt Kessler, NDM Research Building, Target Discovery Institute, Roosevelt Drive, Old Road Campus, Oxford, OX3 7FZ, United Kingdom. E-mail: benedikt.kessler@ndm.ox.ac.uk

\footnotetext{
*These authors contributed equally.

From the ${ }^{1}$ Nuffield Department of Clinical Neurosciences, University of Oxford, United Kingdom; and ${ }^{2}$ Target Discovery Institute, University of Oxford, Oxford, United Kingdom 
with the histopathological finding of ubiquitinated, transactive response DNA binding protein $43 \mathrm{kDa}$ (TDP-43)positive neuronal and oligodendroglial cytoplasmic inclusions, ${ }^{2}$ but the precise cascade of events leading to neuronal death and TDP-43 accumulation is still elusive. The clinical heterogeneity of ALS means there is pressing need for effective biomarkers to help reduce diagnostic delay, stratify, and monitor response in therapeutic trials. ${ }^{3}$ Cerebrospinal fluid (CSF) neurofilament levels are a leading candidate. ${ }^{4}$

Neuroinflammation undoubtedly occurs in ALS, though whether this is pathogenic or a secondary consequence of neurodegeneration is uncertain. In the latter case, anti-inflammatory drug targets may still modify disease progression. Both animal models and postmortem tissue from ALS patients shows infiltration of activated microglia and $\mathrm{T}$ cells into affected areas, which correlates with progression rate. ${ }^{5}$ In vivo microglial activation in affected regions is observed using human ALS positron emission tomography (PET) imaging. ${ }^{6,7}$ Differential expression of cytokines, including factors specifically involved in microglial activation, have been observed in enzyme-linked immunosorbent assay (ELISA)-based studies of CSF from ALS patients. ${ }^{8,9}$

Proteomic methods offer an agnostic approach to biomarker discovery by comparing large numbers of proteins between groups. Several previous studies have used mass spectrometry (MS) to explore the CSF proteome for novel ALS biomarkers, though there has been limited reproducibility between studies because of differing methodologies, particularly regarding sample pooling and complex preanalytical procedures including depletion of abundant proteins. ${ }^{10,11}$ We applied a state-of-the-art high-throughput MS method to enable in-depth characterization of individual longitudinal CSF samples from a large, clinically well-characterized ALS cohort, in comparison to healthy and disease control groups.

\section{Materials and Methods}

\section{Participants and Sampling}

Ethical approval for this study was obtained from South Central Oxford Ethics Committee B (08/H0605/85), NRES Central Committee South Central-Berkshire (14/SC/0083 and 10/ H0505/71). All participants provided written consent (or gave permission for a carer to sign on their behalf). The study included 43 patients with ALS, 6 with the upper motor neuron-only variant of MND, primary lateral sclerosis (PLS), 20 with Parkinson's disease (PD), 12 with ALS mimic conditions (including multifocal motor neuropathy with conduction block and Kennedy's disease), and 25 healthy control subjects. Patients with ALS, PLS, and mimic disorders were recruited from the Oxford ALS Centre (Oxford, UK), and patients with
PD were recruited through the Oxford Parkinson's Disease Centre (Oxford UK). Healthy control subjects were typically spouses and friends of those with ALS, PLS, and mimic disorders. Exclusion criteria included age $<18$ years and/or contraindication to lumbar puncture, including inherited bleeding disorders, chronic liver disease and use of anticoagulant or antiplatelet drugs, and systemic illness at the time of CSF sampling. Control subjects were excluded if there was any family history of ALS or PLS, and ALS and PLS patients were excluded if there was severe physical disability that would prevent communication of distress during lumbar puncture. Patients with ALS, ${ }^{12}$ PLS, ${ }^{13}$ and mimic disorders were diagnosed according to standard criteria by two neurologists experienced in the diagnosis of motor disorders (M.R.T., K.T.). Patients with ALS or PLS were followed until death or censorship (Table 1).

Subjects gave a single CSF sample at each visit, and clinical data were ascertained on the same day. CSF was collected at baseline and, in ALS and PLS patients, every 6 months when available; visits were numbered for each subject sequentially from the baseline visit. CSF samples were processed in accord with consensus guidelines for biomarker development within 1 hour of sampling and stored at $-80^{\circ} \mathrm{C}$ until use. ${ }^{14}$ Symptom onset was defined as first weakness reported by patients. Disease progression rate was calculated per visit using the revised ALS Functional Rating Scale (ALSFRS-R) by [48 - ALSFRS-R]/[months from symptom onset]. Patients were divided into fast and slow progressing using a disease progression rate above or below the median value. A clinical upper motor neuron burden score $(0-15)$ was calculated by the presence of hyper-reflexia. ${ }^{15}$ Demographic and clinical characteristics are displayed in Table 1.

\section{Sample Preparation for Proteomic Analysis}

Samples of CSF were thawed on ice and digested using heatstable immobilized trypsin (SMART digest; Thermo Fisher Scientific, Loughborough, UK). CSF $(50 \mu \mathrm{l})$ was mixed with $150 \mu \mathrm{l}$ of SMART digest buffer and added to SMART digest plates. Samples were incubated at $70^{\circ} \mathrm{C}$ with shaking at $1,400 \mathrm{rpm}$ for 60 minutes. Digested samples were desalted using SOLA $\mu$ plates and dried by vacuum centrifugation. Samples were resuspended in $20 \mu \mathrm{l}$ buffer A ( $2 \%$ acetonitrile, $0.1 \%$ formic acid in water) and kept at $-20^{\circ} \mathrm{C}$ until analysis. Peptide concentrations were assayed using a Pierce (Thermo Fisher Scientific, UK) quantitative colorimetric peptide assay according to the manufacturer's instructions. A pooled sample was produced by combining equal quantities of digested peptide from each individual sample and injected after every 10th sample for use in qualitycontrol analysis.

\section{Proteomic Analysis}

Peptides were analyzed by nano ultra-high performance liquid chromatography tandem mass spectrometry (nUHPLC LC-MS/ MS) using a Dionex Ultimate 3000 UHPLC (Thermo Fisher Scientific, Schwerte, Germany) coupled to a Q Exactive HF tandem mass spectrometer (Thermo Fisher Scientific, Germany). Peptides (500nl) from each sample were injected and analyzed using a 60-minute linear gradient at a $250-\mathrm{nl} /$ minute 
TABLE 1. Demographic Data

\begin{tabular}{|c|c|c|c|c|c|c|}
\hline & ALS & PLS & $\begin{array}{l}\text { Healthy } \\
\text { Control }\end{array}$ & $\begin{array}{l}\text { Parkinson's } \\
\text { Disease }\end{array}$ & $\begin{array}{l}\text { Mimic } \\
\text { Conditions }\end{array}$ & $p$ \\
\hline $\mathrm{n}$, first visit & 43 & 6 & 20 & 20 & 12 & - \\
\hline $\mathrm{n}$, second visit & 21 & 5 & - & - & - & - \\
\hline $\mathrm{n}$, third visit & 13 & 3 & - & - & - & - \\
\hline $\mathrm{n}$, fourth visit & 10 & 2 & - & - & - & - \\
\hline $\mathrm{n}$, fifth visit & 2 & - & - & - & - & - \\
\hline$\%$ male & 72.1 & 16.7 & 55.0 & 50.0 & 91.7 & $<0.001$ \\
\hline $\begin{array}{l}\text { Age at sampling, } \\
\text { years, mean } \pm S D\end{array}$ & $61.9 \pm 10.3$ & $67.98 \pm 6.90$ & $58.5 \pm 8.6$ & $62.58 \pm 4.10$ & $57.7 \pm 15.5$ & 0.175 \\
\hline $\begin{array}{l}\text { Age at onset, } \\
\quad \text { years, mean } \pm S D\end{array}$ & $59.32 \pm 10.9$ & $40.72 \pm 34.40$ & - & $60.83 \pm 4.00$ & - & 0.673 \\
\hline$\%$ bulbar onset & 18.60 & 16.67 & - & - & - & 0.140 \\
\hline $\begin{array}{l}\text { Median disease } \\
\text { progression rate (Q1, Q3) }\end{array}$ & $\begin{array}{l}0.50 \\
(0.28-0.95)\end{array}$ & $\begin{array}{l}0.12 \\
(0.09-0.15)\end{array}$ & - & - & - & $<0.001$ \\
\hline $\begin{array}{l}\text { Duration of follow-up, } \\
\text { months, mean } \pm S D\end{array}$ & $34.0 \pm 26.5$ & $80.0 \pm 15.4$ & - & - & - & $<0.001$ \\
\hline No. of deaths (\%) & $35(81)$ & 0 & - & - & - & $<0.001$ \\
\hline
\end{tabular}

flow rate. The gradient used to elute the peptides started at 3 minutes with $2 \%$ buffer B $(0.1 \%$ trifluoroacetic acid and $5 \%$ dimethyl sulfoxide in $\mathrm{CH}_{3} \mathrm{CN}$ ) increasing to $5 \%$ by 6 minutes followed by an increase up to $35 \%$ by 63 minutes. Data were acquired with a resolution of 60,000 full width at half maximum ion intensity with a mass/charge ratio of 400 and a lockmass enabled at $445.120025 \mathrm{~m} / \mathrm{z}$. The 12 most abundant precursor ions in each MS1 scan were selected for fragmentation by higher-energy collisional dissociation at a normalized collision energy of 28 followed by exclusion for 27 seconds.

Raw MS data were analyzed using Progenesis QI for Proteomics software (v3.0; Nonlinear Dynamics, Newcastle upon Tyne, UK). MS/MS spectra were searched against the UniProt Homo Sapiens Reference proteome (retrieved January 6, 2017) using Mascot (v2.5.1; Matrix Science, Inc., Boston, MA), allowing for a precursor mass tolerance of $10 \mathrm{ppm}$ and a fragment ion tolerance of $0.05 \mathrm{Da}$. Deamidation on asparagine and glutamine and oxidation on methionine were included as variable modifications. The peptide false discovery rate (FDR) was set at $1 \%$, and all peptides with an ion score higher than 20 into were imported into Progenesis QIP. Proteins that were defined with at least one unique peptide were included in the protein data set for further analysis. Protein abundance values were centered to the median abundance of the $90 \%$ of proteins with the lowest variance across all runs and scaled by median absolute deviation. ${ }^{16}$ Missing data were imputed using three nearest neighbors imputation. Non-normalized label-free quantitation data can be found in Supplementary Table 2. Median coefficient of variation (CV) of pooled samples was $12.48 \%$ (interquartile range [IQR], 7.93-23.4) across all protein groups quantified. Median combined coefficient of variation (biological and analytical) across all protein groups in first visit samples from all subjects was $20.11 \%$ (IQR, 14.68-28.82; difference in variation between pools and individual first visit samples, $p<$ 0.001 ), with $74.11 \%$ of proteins having $\mathrm{CV}<25 \%$, giving a statistical power of at least 0.8 for a 1.5 -fold difference in these proteins at FDR-adjusted $p<0.05$ for all comparisons. ${ }^{17}$ Quantification of pooled samples was highly correlated, with median between-pool correlation Pearson's $r=0.94(p<$ 0.001 for all between-pool correlations; IQR, 0.90-0.97).

In relation to the three candidate markers identified, combined variation in first samples across all subjects was $45.86 \%$ for chitotriosidase-1 (CHIT1; combined variation in ALS samples $45.73 \%$, healthy controls $24.39 \%$, PD $25.22 \%$, PLS $30.21 \%$, and mimic $27.19 \%$ ), $16.21 \%$ for chitinase-3-like protein 1 (CHI3L1; combined variation in ALS samples $15.18 \%$, healthy controls $12.67 \%$, PD $12.75 \%$, PLS $3.55 \%$, and mimic $14.32 \%$ ), and $36.8 \%$ for chitinase-3-like protein 2 (CHI3L2; combined variation in ALS samples 38.49\%, healthy controls $21.73 \%$, PD $28.22 \%$, PLS $35.09 \%$, and mimic 
$33.01 \%)$. The within-individual variation across multiple visits for individuals undergoing longitudinal sampling was median 12.52\% (IQR, 7.06-21.87) for CHIT1, 4.79\% (2.70-6.92) for CHI3L1, and 14.27\% (8.97-22.62) for CHI3L2.

To examine changes in the accuracy of quantification across the all samples, normalized log abundances of all protein groups in the first sample were subtracted from the subsequent samples and a linear model created to examine changes in the median deviation of relative abundance of all proteins from the first sample. This did not change significantly over the course of the experiment (gradient $=0.00 ; \log$ abundance units per sample, $p=0.062$ ).

\section{ELISA}

CSF phosphorylated neurofilament heavy chain ( $\mathrm{pNFH}$ ) concentrations were measured according to manufacturer's instructions in duplicate wth a CE-labeled ELISA with a lower detection limit of $270 \mathrm{pg} / \mathrm{ml}$ for pNFH (Euroimmun AG, Lübeck, Germany). CSF samples were assayed neat and the mean intra-assay CV for standard curve measurements was $10.26 \%$ and interassay CV was $10.13 \%$. All samples were measured by the same individual who was blinded to any clinical data.

\section{Statistical Analysis}

Statistical analysis was performed and plots produced using $\mathrm{R}$ (v3.3.3; R core team). ${ }^{18}$ Cross-sectional analysis used first visit samples and data from patients with ALS and PLS. Relative abundance in cross-sectional analysis was compared using a MannWhitney $U$ test with FDR correction using the BenjaminiHochberg step-up procedure, with FDR-adjusted $p<0.05$ (or unadjusted $p<0.05$ where appropriate) used to denote statistical significance. $p$ values are given to three decimal places, other parameters to two decimal places. Receiver operator characteristic (ROC) analysis was performed to assess classifier performance, and ROC curves were compared using the DeLong method (using paired analysis to account for the use of multiple markers in the same samples). ${ }^{19}$ Correlation with clinical parameters was calculated using Pearson's correlation coefficient and tested using the Student's $t$ test. Cross-sectional analysis was performed using data and samples obtained from an individual's first visit. Correlation between chitinase proteins and $\mathrm{pNFH}$ levels was performed using data across all visits. Cox regression analysis of survival was performed incorporating available prognostic covariates and univariate analysis using the log-rank test, again using data from first visit samples. Longitudinal data analysis was performed using a random intercept, random slope linear mixed model.

\section{Results}

CHIT1, CHI3L1, and CHI3L2 Are Elevated in ALS Seven hundred seventy-three protein groups were identified and quantified across conditions with at least one unique peptide. Nineteen protein groups were differentially abundant with FDR-adjusted $p<0.05$ (Supplementary Table 1), three between ALS and healthy controls, 16 between ALS and PD, and one between ALS and mimic disorders. No proteins were differentially abundant with FDR-adjusted $p<0.05$ between ALS and PLS (Fig 1).

The proteins with FDR-adjusted $p<0.05$ between ALS and healthy controls comprised three chitinases produced by cells of the immune system, particularly macrophages: chitotriosidase (CHIT1), chitinase-3-like protein 1 $(\mathrm{CHI} 3 \mathrm{~L} 1)$, and chitinase-3-like protein 2 (CHI3L2). ${ }^{20}$ All three proteins were elevated in ALS compared to other groups except PLS. CSF levels of CHI3L1 did not differ significantly between ALS and PLS ( $\log _{2}$ fold change, 0.04; unadjusted $p=0.666$ ), though elevation of CHIT1 and CHI3L2 were observed in ALS compared to PLS (Fig 2A-C; fold change, unadjusted $p$ value, and FDR-adjusted $p$ for chitinase proteins are given in Table 2 and for all protein groups identified in Supplementary Table 1).

Multivariate ROC analysis was performed to assess the utility of the three markers in differentiating between ALS and mimic samples (using first visit samples only), which represents the most clinically relevant distinction. Each marker performed well (CHIT1 area under the curve [AUC] 0.81, 95\% confidence interval [CI] 0.69-0.93, $p<$ 0.001; CHI3L1 AUC 0.90, CI 0.82-0.98, $p<0.001$; CHI3L2 AUC 0.81, CI 0.68-0.95, $p<0.001$; CHIT1 vs CHI3L1 $p=0.199$, CHIT1 vs CHI3L2 $p=0.962$, CHI3L1 vs CHI3L2 $p=0.286$ ). A combination of all three markers (by summation of centered and scaled log abundance values) provided the best overall performance (AUC, 0.95; CI, 0.88-1.00, $p<0.001$; Fig 2D), improving upon CHIT1 or CHI3L2 performance, but not CHI3L1 (combined vs CHIT1, $p=0.049$; combined vs CHI3L1, $p=0.385$; combined vs CHI3L2, $p=0.009$ )

\section{Chitinase Levels Correlate With Disease Progression Rate}

Regression models were constructed using samples and clinical data obtained at the first visit of patients with ALS and PLS. Using simple linear regression, CHIT1, CHI3L1, and CHI3L2 levels correlated positively with disease progression rate calculated at the first visit (CHIT1 vs log [disease progression rate], Pearson's $r=$ $0.56, p<0.001$; CHI3L1, $r=0.31, p=0.028$; CHI3L2, $r=0.29, p=0.044$; Fig 3A-C). There was no correlation with degree of disability as measured by ALSFRS-R for any of the three chitinase proteins (CHIT1, $r=-0.13$; $p=0.364$; CHI3L1, $r=-0.06$, $p=0.694$; CHI3L2 $r=-0.12$; $p=0.431$; Fig 3D-F). Multiple linear regression models controlling for the effect of age, sex, absolute ALSFRS-R, progression rate, and site of onset (bulbar vs spinal) on chitinase protein level demonstrated significant positive correlation with disease progression rate for CHIT1, CHI3L1, and CHI3L2 (CHIT1, $p<0.001$; gradient $=0.27 \mathrm{log}$ 

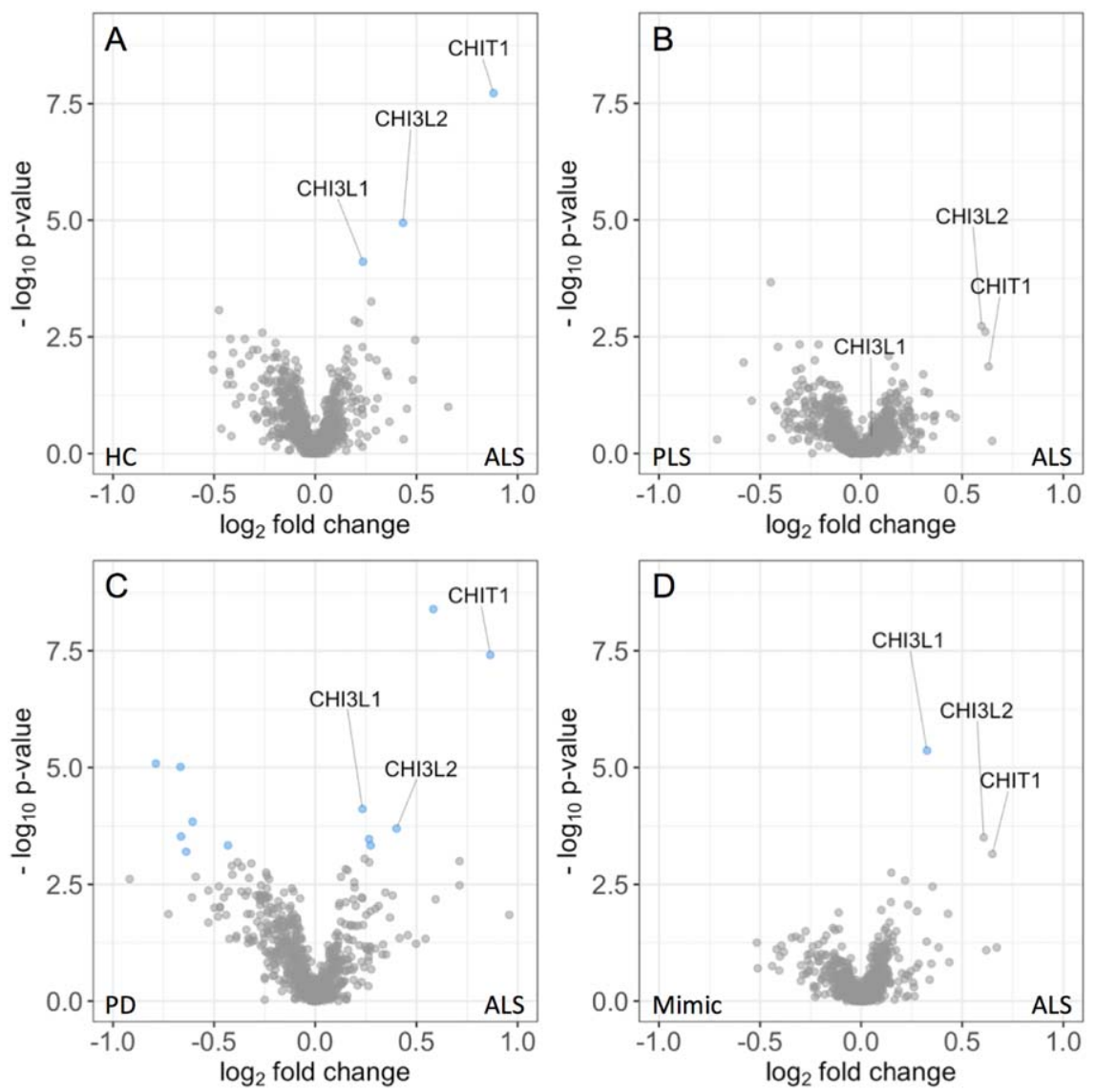

FIGURE 1: "Volcano plot" showing $\log _{2}$ fold change vs unadjusted $p$ values for 773 proteins quantified using LC-MS/MS with label-free quantification. Positive $\log _{2}$ fold change indicates proteins upregulated in ALS. Blue points represent FDR-adjusted $p$ values $<0.05$. Consistently differentially abundant proteins CHIT1, CHI3L1, and CHI3L2 are labeled. (A) ALS vs healthy control subjects. (B) ALS vs the upper motor neuron variant primary lateral sclerosis. (C) ALS vs Parkinson's disease. (D) ALS vs mimic disorders. ALS = amyotrophic lateral sclerosis; $\mathrm{HC}=$ healthy control; PLS = primary lateral sclerosis; PD = Parkinson's disease; CHIT1 = chitotriosidase; CHI3L1 = chitinase-3-like protein 1; CHI3L2 = chitinase-3-like protein 2; FDR = false discovery rate; LC-MS/MS = liquid chromatography with tandem mass spectrometry.

abundance units per unit increase in log disease progression rate; $\mathrm{CHI} 3 \mathrm{~L} 1, p=0.036$; gradient $=0.05 \mathrm{log}$ abundance units per unit increase in log disease progression rate; CHI3L2, $p=0.033$; gradient $=0.13 \log$ abundance units per unit increase in log disease progression rate). CHIT1 and CHI3L2 levels were lower in older subjects (CHIT1, $p=0.025$; gradient $=-0.02$ $\log$ abundance units per year; CHI3L2, $p=0.032$; gradient $=-0.017 \log$ abundance units per year) in crosssectional analysis of patients with ALS and PLS.

Multiple linear regression models were also constructed to explore the contribution of age and sex across the cohort, modeling each chitinase level separately, with age, sex, and subject grouping as explanatory variables; no significant association with age or sex was observed.

\section{CHIT1, CHI3L1, and CHI3L2 Correlate With a Marker of Axonal Damage}

The neurofilament protein, pNFH, is a marker of axonal damage, and multiple studies have shown significant elevation in ALS CSF that also show correlation with disease progression rate (reviewed in $\mathrm{Xu}$ et $\mathrm{al}^{4}{ }^{4}$ ). Levels of CHIT1, CHI3L1, and CHI3L2 were all positively correlated with pNFH levels (CHIT1, Pearson's $r=0.62$, $p<0.001$; CHI3L1, $r=0.49, p<0.001$; CHI3L2, $r=0.41, p<0.001$; Fig 3G-I).

Additional linear regression analysis was performed to model disease progression rate using first visit protein (CHIT1, CHI3L1, CHI3L2, and pNFH) and the clinical variables age at onset, age at sampling, onset site (bulbar vs spinal), and sex. To provide comparable parameter estimates, levels of CHIT1, CHI3L1, CHI3L2, and pNFH were centered about 0 and scaled to standard deviation 1. A model incorporating all explanatory variables showed significant associations of decreasing age and increasing $\mathrm{pNFH}$ with increasing progression rate (model adjusted $R^{2}=0.65, p<0.001$; age of onset gradient $=$ $-0.14 \log$ points per month per year, $p<0.001$; pNFH gradient $=0.69 \log$ points per month per log abundance unit, $p=0.006$; Table 3). The most parsimonious 

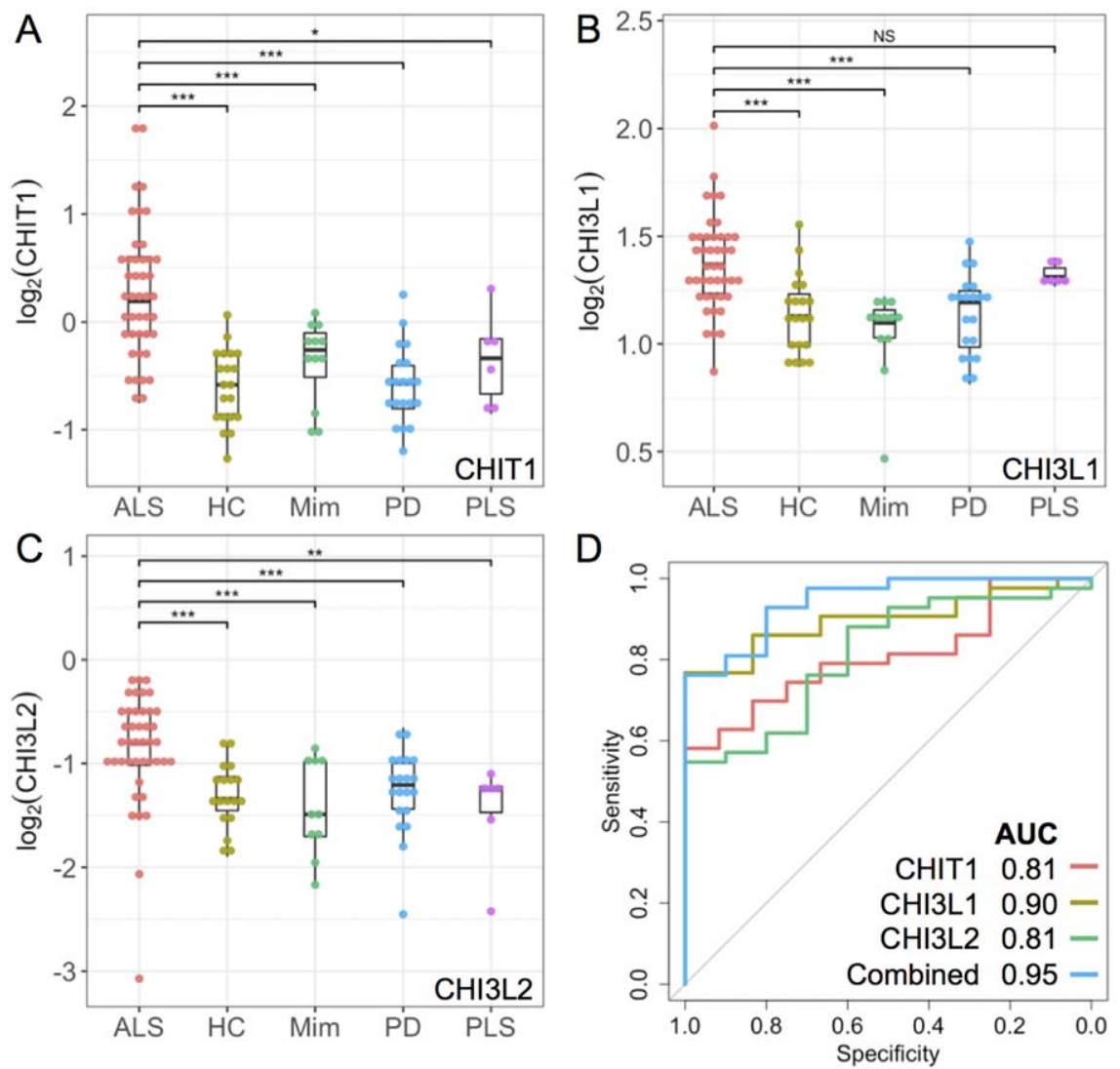

FIGURE 2: Cross-sectional analysis. (A-C) normalized $\log _{2}$ relative abundance of CHIT1, CHI3L1, and CHI3L2 between conditions (first visit samples only). Significance bars indicate unadjusted $p$ values for first visit samples. ${ }^{*} p<0.05$; ${ }^{* *} p<0.01$; ${ }^{\star * \star} p<0.001 ;{ }^{N S} p \geq 0.05$. (D) Performance of CHIT1, CHI3L1, CHI3L2, and combined (summed centered and scaled log abundance) in differentiating ALS from mimic first visit samples by receiver operator characteristic. ALS = amyotrophic lateral sclerosis; $\mathrm{HC}=$ healthy control; Mim = mimic disorders; PLS = primary lateral sclerosis; $\mathrm{PD}=$ Parkinson's disease; $\mathrm{CHIT1}=$ chitotriosidase; CHI3L1 = chitinase-3-like protein 1; CHI3L2 = chitinase-3-like protein 2; AUC = area under the curve.

model of disease progression rate with highest adjusted $R^{2}$ comprised just three explanatory variables: age at symptom onset, CHIT1, and pNFH levels. This demonstrated significant associations of decreasing age, increasing $\mathrm{pNFH}$, and increasing CHIT1 levels with higher disease progression rate (adjusted $R^{2}=0.67$; age at onset, $p<0.001$; gradient $=-0.14 \log$ points per month per year; $\mathrm{pNFH} p=0.005$, gradient $=0.61 \mathrm{log}$ points per month per log abundance unit; $\mathrm{CHIT} 1 p=$ 0.045 , gradient $=0.29$ points per month per log

TABLE 2. Relative Abundance of Chitinase Proteins in Cerebrospinal Fluid From Patients With Amyotrophic Lateral Sclerosis Compared to Healthy Controls and Those With Parkinson's Disease, Primary Lateral Sclerosis, and Mimic Conditions

\begin{tabular}{|c|c|c|c|c|c|c|c|c|c|c|c|c|}
\hline \multirow[b]{2}{*}{ Protein } & \multicolumn{3}{|c|}{$\begin{array}{c}\text { ALS vs Healthy } \\
\text { Control }\end{array}$} & \multicolumn{3}{|c|}{$\begin{array}{c}\text { ALS vs Parkinson's } \\
\text { Disease }\end{array}$} & \multicolumn{3}{|c|}{ ALS vs PLS } & \multicolumn{3}{|c|}{$\begin{array}{l}\text { ALS vs Mimic } \\
\text { Conditions }\end{array}$} \\
\hline & FC & $p$ & FDR & FC & $p$ & FDR & FC & $p$ & FDR & FC & $p$ & FDR \\
\hline Chitotriosidase-1 & 0.87 & $<0.001$ & $<0.001$ & 0.85 & $<0.001$ & $<0.001$ & 0.62 & 0.014 & 0.755 & 0.64 & $<0.001$ & 0.197 \\
\hline Chitinase-3-like protein 2 & 0.43 & $<0.001$ & 0.005 & 0.39 & 0.002 & 0.025 & 0.59 & 0.002 & 0.471 & 0.62 & $<0.001$ & 0.081 \\
\hline Chitinase-3-like protein 1 & 0.23 & $<0.001$ & 0.013 & 0.23 & $<0.001$ & 0.011 & 0.04 & 0.666 & 0.915 & 0.32 & $<0.001$ & 0.004 \\
\hline
\end{tabular}



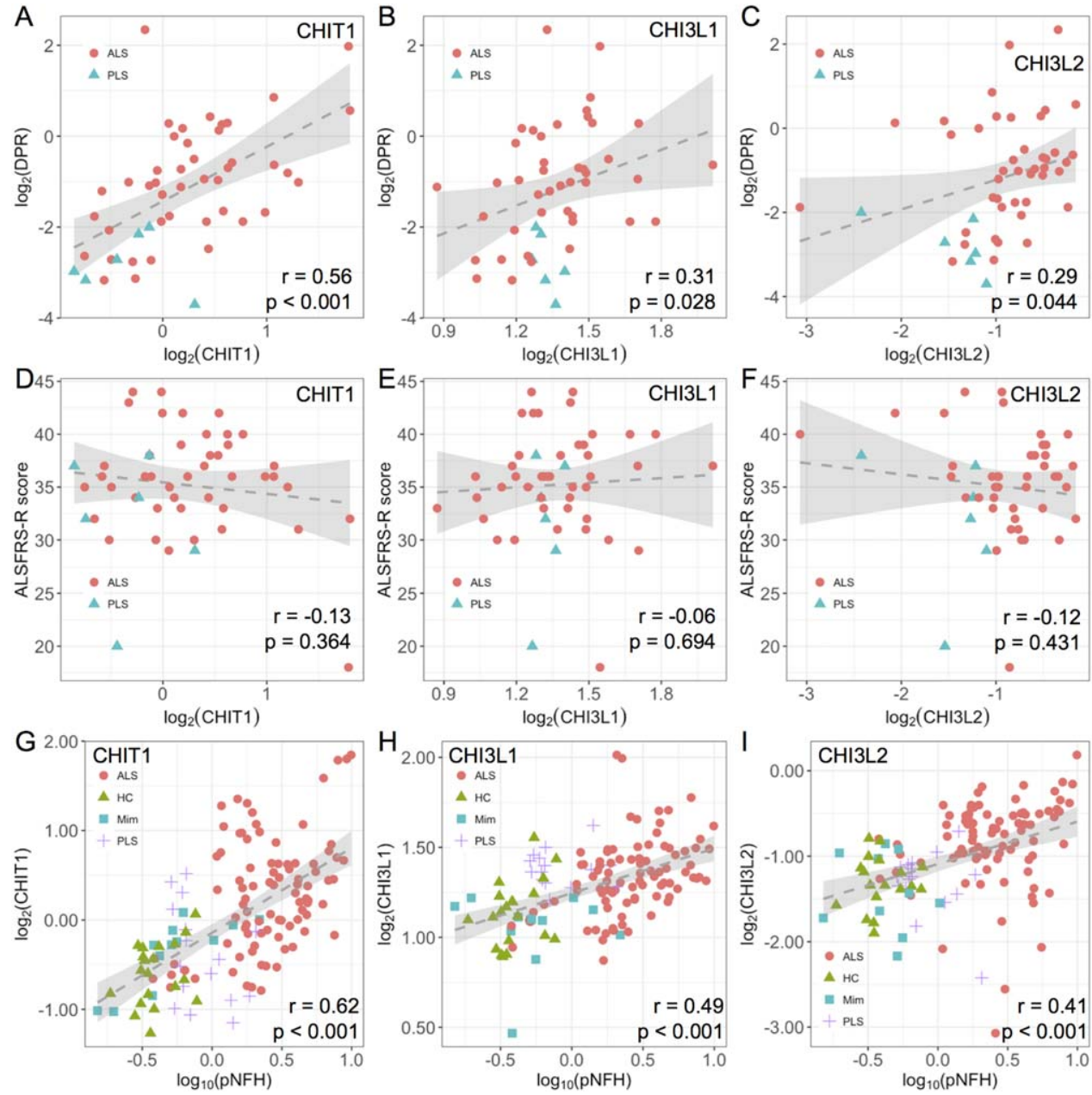

FIGURE 3: Correlation analysis. (A-C) Correlation between CHIT1, CHI3L1, and CHI3L2 and disease progression rate measured by rate of decline in ALSFRS-R (points per month). Ribbon indicates $95 \%$ confidence interval of linear model fit. Pearson's $r$ and $p$ value given for simple linear correlation, first visit samples only. (D-F) Correlation between CHIT1, CHI3L1, and CHI3L2 and disability as measured by ALSFRS-R score. Ribbon indicates $95 \%$ confidence interval of linear model fit. Pearson's $r$ and $p$ value given for simple linear correlation, first visit samples only. (G-I) Correlation between CHIT1, CHI3L1, and CHI3L2 and pNFH. Ribbon indicates $95 \%$ confidence interval of linear model fit. Pearson's $r$ and $p$ value for correlation, all samples. ALS = amyotrophic lateral sclerosis; $\mathrm{HC}=$ healthy control; Mim = mimic disorders; PLS = primary lateral sclerosis; PD = Parkinson's disease; CHIT1 = chitotriosidase; CHI3L1 = chitinase-3-like protein 1; CHI3L2 = chitinase-3-like protein 2; DPR = disease progression rate (ALSFRS-R decline, points per month); ALSFRS-R = revised ALS Functional Rating Scale; pNFH = phosphorylated neurofilament heavy chain.

abundance unit; Table 3), though it does not take account of clinical parameters that have previously been associated with higher disease progression rate.

\section{Longitudinal Analysis}

A linear mixed model of CSF CHIT1, CHI3L1, and CHI3L2 was fitted to longitudinal values with age and time from first sampling as covariates, beginning at the baseline visit, with patients stratified by level at baseline visit in relation to median value. Longitudinal sample numbers for ALS and PLS are summarized in Table 1. There was a small increase in CHI3L1 levels over time in those with low levels at onset (gradient $=0.005 \log$ abundance units per month, $p=0.001$ ). The gradient did not differ significantly from zero in those with high initial CHI3L1 or for either stratum of CHIT1 or CHI3L2 (Fig 4).

\section{Baseline CHIT1 Level, but Not CHI3L1 and CHI3L2, Is Associated With Survival}

A Cox proportional hazards model including first visit ALSFRS-R, age at onset, sex, onset site (bulbar vs spinal), and CHIT1, CHI3L1, and CHI3L2 levels in all MND patients showed association of CHIT1 level and age with survival (CHIT1 hazard ratio [HR] 2.84, $p=0.009,95 \%$ CI 1.24-5.75; CHI3L1 HR $=1.23, p=0.55,95 \% \mathrm{CI}$ 0.64-2.33; CHI3L2 HR $=1.08, p=0.93,95 \%$ CI $0.20-7.13$; age $\mathrm{HR}=1.00015, p=0.008,95 \% \mathrm{CI}$ 1.00004-1.00027; Table 4). The addition of pNFH level to the model leaves only pNFH as an independent prognostic factor $(\mathrm{pNFH}$ HR $=1.26, p=0.019,95 \%$ CI 
TABLE 3. Multiple Linear Regression Modeling of Disease Progression Rate Using Chitinase Proteins, pNFH, and Clinical Parameters

\begin{tabular}{|c|c|c|c|c|c|c|}
\hline & Gradient & $95 \% \mathrm{CI}$ & $p$ & Gradient & $95 \% \mathrm{CI}$ & $p$ \\
\hline Age at onset & -0.13 & -0.15 to -0.06 & $<0.001$ & -0.14 & -0.22 to -0.06 & $<0.001$ \\
\hline pNFH & 0.61 & $0.19-1.04$ & 0.006 & 0.69 & $0.21-1.17$ & 0.006 \\
\hline CHIT1 & 0.29 & $0.01-0.57$ & 0.045 & 0.29 & $-0.04-0.61$ & 0.085 \\
\hline CHI3L1 & - & - & - & -0.10 & $-0.40-0.19$ & 0.487 \\
\hline CHI3L2 & - & - & - & 0.07 & $-0.16-0.30$ & 0.537 \\
\hline Sex (male) & - & - & - & 0.25 & $-0.79-0.29$ & 0.360 \\
\hline Site of onset (limb) & - & - & - & 0.00 & $-0.61-0.61$ & 0.999 \\
\hline Adjusted $R^{2}$ & 0.67 & - & $<0.001$ & 0.65 & - & $<0.001$ \\
\hline
\end{tabular}

1.04-1.53; CHIT1 HR 1.90, $p=0.137,95 \%$ CI $0.82-$ 4.44; CHI3L1 HR $=1.37, p=0.765,95 \%$ CI $0.18-$ 10.60; CHI3L2 HR $=0.88, p=0.728,95 \%$ CI $0.43-$ 1.81). Data for several factors known to influence survival in ALS including nutritional status and respiratory function (ie, forced vital capacity) were not available, so caution is needed in drawing firm conclusions around an independent role for CHIT1 levels at present.

Kaplan-Meier survival curves showed apparent separation of cumulative survival of patients when stratified by level of CHIT1, CHI3L1, or CHI3L2, though no significant association between stratum and survival was observed (log-rank test, CHIT1 $p=0.18, \mathrm{CHI} 3 \mathrm{~L} 1 p=$ 0.08, CHI3L2 $p=0.15$; Fig 5).

\section{Discussion}

This proteomic discovery study has demonstrated elevation of three mammalian chitinases in CSF from ALS patients compared to relevant controls. Unique strengths include the relatively large number of individual-level patient samples analyzed without the confound of sample pooling; a high-throughput approach designed to minimize technical variability and the availability of longitudinal CSF samples

Elevation of CSF CHIT1, CHI3L1 and CHI3L2 has been previously demonstrated in a single small proteomic study of samples pooled from 10 ALS patients, ${ }^{11}$ and a larger ELISA-based study further confirmed the localization of central nervous system (CNS) CHIT1 expression to microglia. ${ }^{21}$
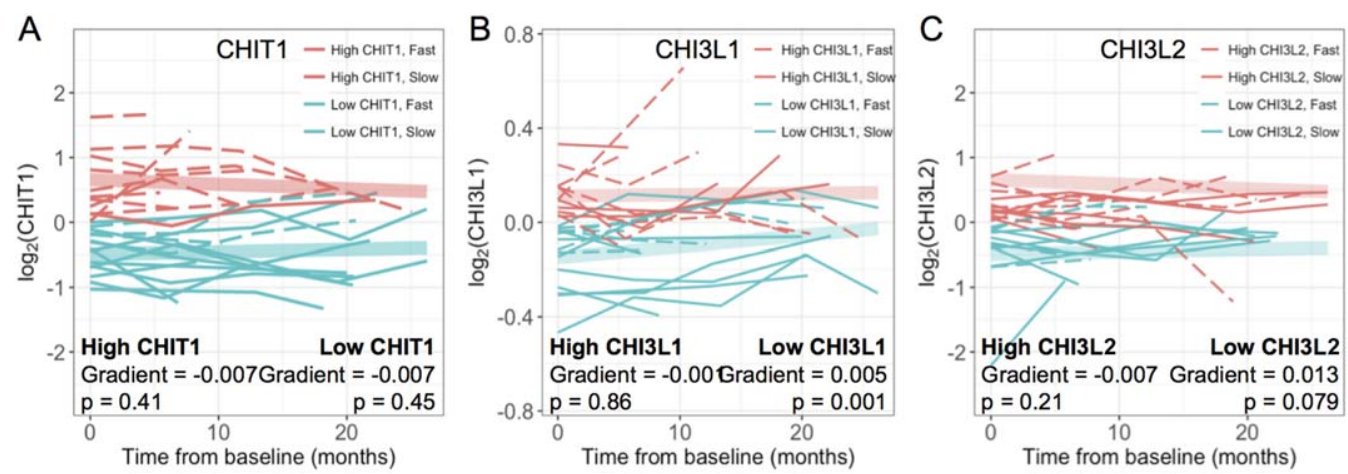

FIGURE 4: (A-C) Longitudinal stability of CHIT1, CHI3L1, and CHI3L2. Gradient estimated using a random slope, random intercept linear mixed model, stratified by initial analyte level. Ribbon indicates mean predicted longitudinal change by stratum. Gradient in $\log _{2}$ units change per month; $p$ value denotes likelihood that gradient differs from zero. Patients stratified by disease progression rate at last visit. $\mathrm{CHIT1}=$ chitotriosidase; $\mathrm{CHI} 3 \mathrm{~L} 1$ = chitinase-3-like protein 1 ; $\mathrm{CHI} 3 \mathrm{~L} 2=$ chitinase-3-like protein 2. 


\begin{tabular}{|c|c|c|c|c|c|c|}
\hline & HR & $95 \% \mathrm{CI}$ & $p$ & HR & $95 \% \mathrm{CI}$ & $p$ \\
\hline Age at onset & 1.00 & $1.00004-1.00027$ & 0.008 & 1.00 & $1.00000-1.00019$ & 0.149 \\
\hline Sex (male) & 1.32 & $0.53-2.57$ & 0.488 & 0.96 & $0.41-2.21$ & 0.916 \\
\hline ALSFRS- $\mathrm{R}$ at baseline & 1.08 & $0.97-1.18$ & 0.141 & 1.04 & $0.95-1.15$ & 0.359 \\
\hline Site of onset (limb) & 0.74 & $0.30-2.11$ & 0.531 & 0.67 & $0.26-1.70$ & 0.396 \\
\hline CHIT1 & 2.84 & $1.24-5.75$ & 0.009 & 1.90 & $0.82-4.40$ & 0.137 \\
\hline CHI3L1 & 1.23 & $0.64-2.33$ & 0.549 & 1.37 & $0.18-10.60$ & 0.765 \\
\hline CHI3L2 & 1.08 & $0.20-7.13$ & 0.935 & 0.89 & $0.43-1.81$ & 0.728 \\
\hline pNFH & - & - & - & 1.26 & $1.04-1.53$ & 0.019 \\
\hline
\end{tabular}

ALS = amyotrophic lateral sclerosis; PLS = primary lateral sclerosis; $\mathrm{pNFH}=$ phosphorylated neurofilament heavy chain; ALSFRS-R = revised ALS Functional Rating Scale; CHIT1 = chitotriosidase; CHI3L1 = chitinase-3-like protein 1; CHI3L2 = chitinase-3-like protein 2; HR = hazard ratio; $\mathrm{CI}=$ confidence interval.

All three proteins are primarily released by activated macrophages during the host immune response; CHIT1 has been shown to be released by activated microglia in vitro $^{11}$ and colocalize with microglial markers in the spinal cord of patients with ALS. ${ }^{21}$ CHIT1 is an active chitinase, whereas $\mathrm{CHI} 3 \mathrm{~L} 1$ and $\mathrm{CHI} 3 \mathrm{~L} 2$ do not possess chitinase activity, but have broad-ranging roles in the immune response and tissue remodeling including migration of macrophages and glial cells. ${ }^{20}$ CHI3L1 and CHI3L2 expression is elevated in motor cortex and spinal cord of patients with ALS, though the precise cellular origin of this has not been demonstrated. ${ }^{22}$ Given that microglia are the most abundant CNS macrophage and that increased microglial activation is observed in ALS, ${ }^{5,23}$ it seems most likely that our observations relate to microglial activity, though a contribution from other CNS macrophages (namely meningeal and perivascular macrophages) or overspill from the systemic circulation cannot be excluded. Furthermore, because no additional data are available regarding CNS inflammation in this cohort, such as analysis of postmortem tissue or microglial imaging data, the cellular origin of CSF chitinases cannot be categorically demonstrated in this study.

Although it is known that CHIT1, CHI3L1, and CHI3L2 are all expressed by classically activated macrophages, little is known about their relative expression in pro- or anti-inflammatory conditions. ${ }^{24}$ Elevations in CHIT1 have also been observed in lysosomal storage diseases $^{25}$ and multiple sclerosis, ${ }^{26}$ whereas increased CSF CHI3L1 level is associated with progression to multiple sclerosis following a clinically isolated syndrome. ${ }^{27}$

The three markers performed well at classifying ALS versus mimic samples in this cohort, particularly in combination, with AUC 0.95 (95\% CI, 0.88-1.00).
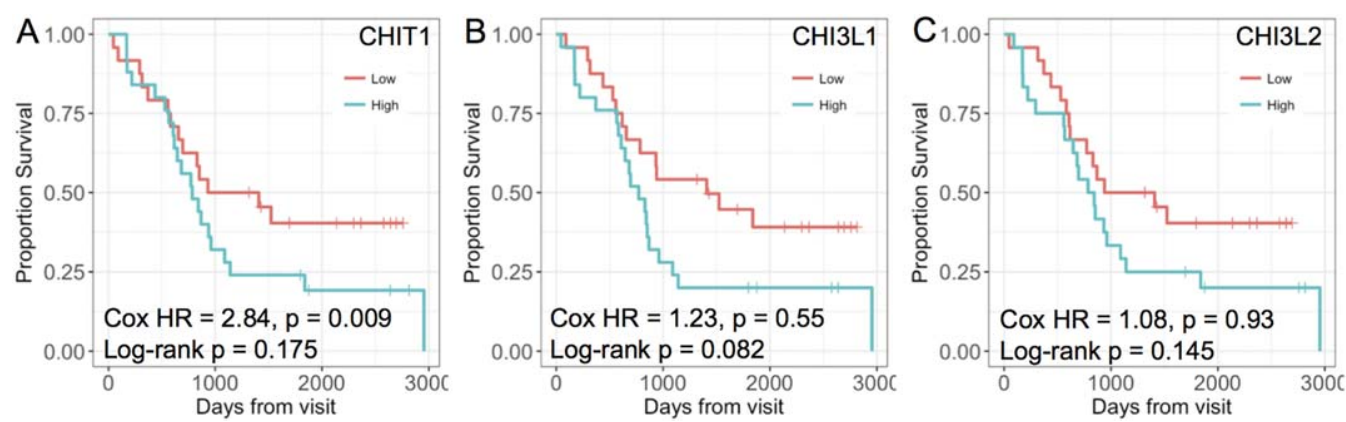

FIGURE 5: (A-C) Survival analysis in ALS and PLS patients from time of first sampling, stratified by relative abundance of CHIT1, CHI3L1, and CHI3L2 at first visit. Cox proportional hazards model hazard ratio (HR) and $p$ value listed along with $p$ value for log-rank test. $\mathrm{CHIT1}=$ chitotriosidase; $\mathrm{CHI}$ L1 = chitinase-3-like protein 1; CHI3L2 = chitinase-3-like protein 2; HR = hazard ratio. 
Although differentiating ALS from ALS-mimic conditions is clinically relevant, ascertainment of the diagnostic accuracy of this set of proteins would require validation in an independent cohort, preferably comprising samples acquired before establishing the definitive diagnosis, from patients in whom the diagnosis of ALS is considered.

Although CHIT1 and CHI3L2 were elevated in ALS compared to all other conditions examined (including PLS), CHI3L1 levels appeared similar in patients with both ALS and PLS. The relationship between ALS and PLS is strongly debated, given their starkly differing natural history, and with no certainty yet in relation to TDP43 because of few postmortem studies in PLS (discussed in Kolind et $\mathrm{al}^{28}$ ). Studying PLS is made more difficult by its rarity, comprising less than 3\% of MND cases and the diagnostic requirement for symptoms to have been present for more than 4 years. This inevitably leads to differences in latency from symptom onset to sampling compared to the ALS group. Given that there was over $80 \%$ power to detect a 1.5 -fold change with $\mathrm{n}=6$ for the majority of proteins (including $\mathrm{CHI} 3 \mathrm{~L} 1),{ }^{17}$ we explored differences at the group level, but recognize the need for caution in drawing any firm conclusions given the small number and the longitudinal increase in CHI3L1 levels in those with a low level at first sampling.

Notwithstanding these caveats, our study hints at potential difference in the microglial response between the two conditions, which might then reflect the striking difference in clinical upper versus lower motor neuron involvement and the rate of progression of symptoms. Regardless of the specific mechanism, differences in the pattern of expression of the three chitinases provide a potential biochemical means to differentiate between ALS and PLS (ie, elevation of CHIT1, CHI3L1, and CHI3L2 in ALS, but only elevation in CHI3L1 in PLS). However, given the limitations outlined, this finding needs additional validation, preferably using orthogonal methods, in a prospective independent cohort before further conclusions can be drawn.

Longitudinal analysis did not demonstrate increasing chitinase levels through the disease course, except for a small increase in CHI3L1 levels in those with a low initial level. This finding does not support a model of disease whereby increased microglial activation drives neurodegeneration, which, in turn, drives further microglial activation, given that, in such a model, longitudinal increases in chitinase proteins would be expected in those with an initially higher level. However, given the small sample size and longitudinal variability of chitinase levels, this cannot be construed as good evidence against such a model.

Significant questions remain as to whether microglial activity influences the rate of disease progression and survival in ALS that is independent of the neurodegenerative process, and the utility of chitinase proteins in measuring this process and predicting survival and disease activity. The regression and survival models presented here do not identify a robust association of CSF chitinase proteins and disease progression rate or survival that is independent of $\mathrm{pNFH}$ and other clinical parameters, and the associations observed (principally with CHIT1) suggest that any association would be smaller in magnitude than that of $\mathrm{pNFH}$ with disease progression rate and survival. Further work to clarify the precise relationship between CSF chitinase levels and CNS microglial activity, along with orthogonal validation of the findings of this study, might help clarify this and further elucidate the relative contribution of neuronal degeneration and neuroinflammation in ALS progression.

As well as complementing measures of axonal damage such as neurofilaments, these proteins represent promising target engagement or pharmacodynamic biomarkers for treatments focused on neuroinflammatory pathways in ALS, or might predict response to proposed therapy. Measurement of CHIT1, CHI3L1, and CHI3L2 alongside in vivo measures of microglial activation, including PET and broader cytokine levels, might further elucidate the role of these chitinase family proteins in ALS pathogenesis.

\section{Acknowledgment}

R.F. and B.M.K. are supported by the Kennedy Trust Fund. M.R.T. is funded by the Medical Research Council \& Motor Neurone Disease Association Lady Edith Wolfson Senior Clinical Fellowship MR/K01014X/1. The Oxford Parkinson's Disease Centre Discovery Cohort is supported by the Monument Trust Discovery Award from Parkinson's UK.

We thank Dr Andreas Jeromin (Iron Horse Diagnostics) and Dr Brita Brix (Euroimmun) for facilitating access to the $\mathrm{pNfH}$ assay as part of a separate dedicated study.

\section{Author Contributions}

All authors contributed to the conception and design of the study. A.G.T., E.G., M.L.T., P.D.C., R.F., and M.R.T. contributed to the acquisition and analysis of data. A.G.T. and E.G. contributed to drafting the text and preparing the figures.

\section{Potential Conflicts of Interest}

Nothing to report.

\section{References}

1. Kiernan MC, Vucic $S$, Cheah BC, et al. Amyotrophic lateral sclerosis. Lancet 2011;377:942-955. 
2. Neumann M, Sampathu DM, Kwong LK, et al. Ubiquitinated TDP43 in frontotemporal lobar degeneration and amyotrophic lateral sclerosis. Science 2006;314:130-133.

3. Turner MR, Benatar M. Ensuring continued progress in biomarkers for amyotrophic lateral sclerosis. Muscle Nerve 2015;51:14-18.

4. Xu Z, Henderson RD, David M, McCombe PA. Neurofilaments as biomarkers for amyotrophic lateral sclerosis: a systematic review and meta-analysis. PLoS One 2016;11:e0164625.

5. Brettschneider J, Toledo JB, Van Deerlin VM, et al. Microglial activation correlates with disease progression and upper motor neuron clinical symptoms in amyotrophic lateral sclerosis. PLoS One 2012;7:e39216

6. Turner MR, Cagnin A, Turkheimer FE, et al. Evidence of widespread cerebral microglial activation in amyotrophic lateral sclerosis: an [11C](R)-PK11195 positron emission tomography study. Neurobiol Dis 2004;15:601-609.

7. Alshikho MJ, Zurcher NR, Loggia ML, et al. Glial activation colocalizes with structural abnormalities in amyotrophic lateral sclerosis. Neurology 2016;87:2554-2561.

8. Mitchell RM, Freeman WM, Randazzo WT, et al. A CSF biomarker panel for identification of patients with amyotrophic lateral sclerosis. Neurology 2009;72:14-19.

9. Rentzos M, Rombos A, Nikolaou $C$, et al. Interleukin-17 and interleukin-23 are elevated in serum and cerebrospinal fluid of patients with ALS: a reflection of Th17 cells activation? Acta Neurol Scand 2010;122:425-429.

10. Collins MA, An J, Hood BL, Conrads TP, Bowser RP. Label-free LC-MS/MS proteomic analysis of cerebrospinal fluid identifies protein/pathway alterations and candidate biomarkers for amyotrophic lateral sclerosis. J Proteome Res 2015;14:4486-4501.

11. Varghese AM, Sharma A, Mishra P, et al. Chitotriosidase-a putative biomarker for sporadic amyotrophic lateral sclerosis. Clin Proteomics 2013;10:19.

12. Brooks BR, Miller RG, Swash M, Munsat TL, World Federation of Neurology Research Group on Motor Neuron D. El Escorial revisited: revised criteria for the diagnosis of amyotrophic lateral sclerosis. Amyotroph Lateral Scler Other Motor Neuron Disord 2000;1:293-299.

13. Gordon $\mathrm{PH}$, Cheng $\mathrm{B}, \mathrm{Katz} \mathrm{IB}$, et al. The natural history of primary lateral sclerosis. Neurology 2006;66:647-653.

14. Teunissen CE, Tumani H, Engelborghs S, Mollenhauer B. Biobanking of CSF: international standardization to optimize biomarker development. Clin Biochem 2014;47:288-292.

15. Menke RA, Korner S, Filippini N, et al. Widespread grey matter pathology dominates the longitudinal cerebral MRI and clinical landscape of amyotrophic lateral sclerosis. Brain 2014;137(pt 9): 2546-2555.
16. Keilhauer EC, Hein MY, Mann M. Accurate protein complex retrieval by affinity enrichment mass spectrometry (AE-MS) rather than affinity purification mass spectrometry (AP-MS). Mol Cell Proteomics 2015;14:120-135.

17. Levin $Y$. The role of statistical power analysis in quantitative proteomics. Proteomics 2011:11:2565-2567.

18. R Core Team. R: A language and environment for statistical computing. 3.2.3 ed. Vienna, Austria: R Foundation for Statistica Computing; 2015.

19. DeLong ER, DeLong DM, Clarke-Pearson DL. Comparing the areas under two or more correlated receiver operating characteristic curves: a nonparametric approach. Biometrics 1988;44 837-845.

20. Di Rosa M, Distefano G, Zorena K, Malaguarnera L. Chitinases and immunity: ancestral molecules with new functions. Immunobiology 2016;221:399-411.

21. Steinacker $P$, Verde F, Fang $L$, et al. Chitotriosidase (CHIT1) is increased in microglia and macrophages in spinal cord of amyotrophic lateral sclerosis and cerebrospinal fluid levels correlate with disease severity and progression. J Neurol Neurosurg Psychiatry. 2017 Nov 15. pii: jnnp-2017-317138. doi: 10.1136/jnnp2017-317138. [Epub ahead of print]

22. Sanfilippo C, Longo A, Lazzara F, et al. CHI3L1 and CHI3L2 overexpression in motor cortex and spinal cord of sALS patients. Mo Cell Neurosci 2017;85:162-169

23. Perry $\mathrm{VH}$, Teeling J. Microglia and macrophages of the central nervous system: the contribution of microglia priming and systemic inflammation to chronic neurodegeneration. Semin Immunopathol 2013;35:601-612.

24. Di Rosa M, Malaguarnera G, De Gregorio C, Drago F, Malaguarnera L. Evaluation of CHI3L-1 and CHIT-1 expression in differentiated and polarized macrophages. Inflammation 2013;36: 482-492.

25. Hollak CE, van Weely S, van Oers MH, Aerts JM. Marked elevation of plasma chitotriosidase activity. A novel hallmark of Gaucher disease. J Clin Invest 1994;93:1288-1292.

26. Mollgaard M, Degn M, Sellebjerg F, Frederiksen JL, Modvig S. Cerebrospinal fluid chitinase-3-like 2 and chitotriosidase are potential prognostic biomarkers in early multiple sclerosis. Eur $\mathrm{J}$ Neurol 2016:23:898-905.

27. Comabella M, Fernandez M, Martin R, et al. Cerebrospinal fluid chitinase 3-like 1 levels are associated with conversion to multiple sclerosis. Brain 2010;133(pt 4):1082-1093

28. Kolind S, Sharma R, Knight S, et al. Myelin imaging in amyotrophic and primary lateral sclerosis. Amyotroph Lateral Scler Frontotemporal Degener 2013;14:562-573. 